Complementary

Medicine Research

\section{Journal Club}

Complement Med Res 2021;28:284-290

DOI: $10.1159 / 000518134$

\title{
Ethno ...
}

Ethnobotanik, Ethnopharmakologie, Ethnomedizin, Ethnoveterinärmedizin - vermutlich ist diese Liste unvollständig. Gemeinsam ist all diesen Forschungsfeldern die wissenschaftliche Auseinandersetzung mit Wissen und Tätigkeiten von Menschen, die aus einer gewissen Erfahrung entspringen. Es bleibt dabei manchmal unklar, ob diese Erfahrung einer langen vielleicht seit vielen Generationen mündlich übertragenen Tradition entspringt oder einer (immer wieder) neuen Erfahrung einzelner Menschen [1, 2]. Nichtsdestotrotz, die oben genannten Forschungsgebiete sind eine wesentliche Wissens- und Ideenbasis der modernen Phytotherapie, sofern die medizinische Intention des Einsatzes von sekundärstoffreichen Pflanzen oder Arzneipflanzen dokumentiert wird.

Traditionelle Ansätze zur Behandlung der Afrikanischen Schlafkrankheit Traditionelle Medizinsysteme sind weltweit in vielen Regionen oft die einzige medizinische Möglichkeit auf die breite Anteile der Bevölkerung Zugriff haben. Die WHO hat dem in ihrer «WHO traditional medicine strategy: 2014-2023» [3] Rechnung getragen. Gleich im ersten Kommentar hat Andreas Lardos dieses aufgegriffen und kommentiert eine Studie aus Angola, die traditionelle Behandlungen der Afrikanischen Schlafkrankheit dokumentiert. Diese über Tsetsefliegen übertragene Krankheit kann sowohl Menschen als auch andere Säugetiere betreffen und ist damit ein Paradebeispiel für «One Health». Behandlungsansätze sind in Human- und Tiermedizin gleichsam willkommen.

Eine süsse Pflanze aus dem Norden - die Echte Engelwurz

Neben der Erfassung des aktuellen Wissens um medizinische Einsatzmöglichkeiten von Pflanzen ist auch die detaillierte Aufbereitung historischer Quellen von grossem Wert. Maja Dal Cero kommentiert eine aktuelle Übersichtsarbeit zur Echten Engelwurz (Angelica archangelica L.) - einer Pflanze, die kulturell und historisch immer an der Grenze zwischen Medizin und Ernährung wandelte und zwischenzeitlich fast in Vergessenheit geriet. Besonders interessant: während viele der heute allgemein bekannten europäischen Arzneipflanzen eher aus mediterranen Regionen stammen, ist die Echte Engelwurz ein echtes Nordlicht. Inwieweit beeinflusst der Sinneseindruck, den eine wilde Pflanze beim Menschen hinterlässt, deren Verwendung und inwieweit beeinflusst der Mensch durch Züchtung die Sinneseindrücke von Pflanzen, die er domestiziert und kultiviert? Bei der Echten Engelwurz steht hier eindeutig «süss» im Vordergrund, aber das ist beileibe nicht alles. 


\section{Nachhaltigkeit traditioneller Medizinsysteme}

Der Mensch kann, wie bei der Echten Engelwurz, zu einer erheblichen Verbreitung einer Pflanzenart beitragen, genauso aber auch Pflanzen bis zur Ausrottung übernutzen. Anhand einer Publikation zur traditionellen Praxis der Substitution von Zutaten in der traditionellen tibetischen Medizin diskutiert Franz Huber dieses spezielle Innovationspotential der Phytotherapie. Nicht zuletzt könnte dieses auch dem Artenschutz zugutekommen.

Hirten am Fusse des Himalayas

Am Ende des heutigen Journal Clubs hatte ich selbst noch die Freude in die Tastatur meines Computers greifen zu dürfen. Im Zentrum steht eine Arbeit zum traditionellen Einsatz von Arzneipflanzen durch Hirten in Kaschmir im Norden Pakistans. Interessanterweise weisen auch die Autor*innen dieser Studie explizit auf den Gefährdungsgrad der einzelnen ethnoveterinärmedizinisch eingesetzten Pflanzen hin. So ist die Indische Kostuswurzel eine offensichtlich sehr häufig eingesetzte Arzneipflanze dieser Region und gleichsam sehr gefährdet. Vielleicht wäre ja auch hier die gezielte Kultivierung oder der Ansatz der Substitution dieser Pflanze durch eine andere ein Ausweg?

\section{Zum guten Schluss}

Die interessantesten und diversesten Ökosysteme findet man immer da, wo unterschiedliche Lebensräume aufeinandertreffen, z.B. in den Mangrovenwäldern (Meer und Festland, Süss- und Salzwasser) oder am Waldrand (Wald und Wiese). Die Diversität wiederum ist, aufgrund ihrer vielen Möglichkeiten ein Garant für die Stabilität eines Gesamtsystems. Auch wenn unterschiedliche Disziplinen zusammenwirken entsteht eine ganz besonders interessante Form inhaltlicher Diversität. In der SMGP entsteht diese Vielfalt im Zusammenklang der Disziplinen Pharmazie, Medizin und Tiermedizin und ist seit vielen Jahren gelebte und belebende Realität. Aber die SMGP ist sogar noch etwas vielfältiger ...

Die heutige Ausgabe des Journal Clubs wurde durch Mitglieder der SMGP gestaltet, die hinsichtlich ihres beruflichen Hintergrunds überwiegend zu den «raren Spezies» innerhalb der SMGP zählen. Andreas Lardos ist Pharmazeut und Biologe, leitet die Fachgruppe Naturstoffchemie und Phytopharmazie an der ZHAW in Wädenswil und ist dort quasi der Nachnachfolger unseres langjährigen Geschäftsstellenleiters Beat Meier. Franz Huber ist Botaniker, Statistiker, hat Sinologie studiert und leitet seit dem vergangenen Jahr die Geschäftsstelle der SMGP. Maja Dal Cero ist Umweltnaturwissenschaftlerin und Ethnobotanikerin, war bis vor kurzem Schatzmeisterin und leitet seit vielen Jahren botanische Exkursionen der SMGP. Was uns alle vier verbindet ist der enge wissenschaftliche Bezug zu «Ethno-PhytoThemen». Freuen Sie sich also auf neue anregende Blickwinkel auf und interessante Schlussfolgerungen für die Phytotherapie.

Michael Walkenhorst, Frick

\section{Literatur}

1 Leonti M. The future is written: impact of scripts on the cognition, selection, knowledge and transmission of medicinal plant use and its implications for ethnobotany and ethnopharmacology. J Ethnopharmacol. 2011 Apr 12;134(3):542-55. doi: 10.1016/j.jep.2011.01.017.

2 Walkenhorst M. Neues aus der Veterinärmedizin, Complement Med Res. 2019, doi: 10.1159/000503909.

3 WHO traditional medicine strategy: 2014-2023. https://apps.who.int/iris/handle/10665/92455. 


\section{Vahekeni N, Neto PM, Kayimbo MK, Mäser P, Josenando T, da Costa E, Falquet J, van Eeuwijk P. Use of herbal remedies in the management of sleeping sickness in four northern provinces of Angola. J Ethnopharmacol. 2020;256:112382.doi 10.1016/j.jep.2019.112382.}

\section{Kommentar}

Andreas Lardos, Wädenswil

In den ärmeren Ländern des Südens ist nach wie vor die Mehrheit der Menschen für ihre primäre medizinische Versorgung auf traditionelle Medizin angewiesen. Deshalb kommt der Sicherheit und der Wirksamkeit von Heilmitteln aus traditionellen oder lokalen Wissenssystemen eine besondere Bedeutung zu. Vor diesem Hintergrund hat die WHO mit ihrer Traditional Medicine Strategy 2014-2023 die Mitgliedstaaten dazu aufgerufen, ihre traditionelle Medizin zu fördern und in die nationalen Gesundheitssysteme zu integrieren, sowie die Qualität der entsprechenden Therapiemassnahmen $\mathrm{zu}$ verbessern. Valide Lösungsansätze aus der traditionellen Medizin sind besonders auch im Bereich der sogenannten vernachlässigten tropischen Krankheiten (neglected tropical diseases) wünschenswert. Dazu gehören verschiedene von Pathogenen ausgelöste Infektionskrankheiten, die im Vergleich zu anderen Gesundheitsproblemen weniger Forschungsförderung und Aufmerksamkeit erhalten. Parasitäre Erkrankung dieser Gruppe sind in Afrika endemisch, darunter auch die Afrikanische Schlafkrankheit (Human African Tropanosomiasis). Diese Erkrankung kommt südlich der Sahara vor, in den Verbreitungsgebieten der Tsetsefliege (Glossina spp.), durch welche der Erreger auf Säugetiere bzw. den Menschen übertragen wird. Zwei Unterarten des verantwortlichen einzelligen Parasiten Trypanosoma brucei sind humanpathogen.

In der vorliegenden Arbeit berichten Vahekeni et al. 2020 über die erstmalige Durchführung einer ethnopharmakologischen explorativen Studie zur Verwendung von traditioneller Medizin für die Behandlung der Afrikanischen Schlafkrankheit im nördlichen Angola. Die Studie verfolgte drei Hauptziele: 1) Ermittlung des Stellenwerts von traditioneller Medizin bei der Behandlung der Erkrankung in vier Provinzen des Landes, in denen diese Erkrankung endemisch ist; 2) Dokumentation der dabei verwendeten Arzneipflanzen; 3) Literatur-Recherche zu Wirksamkeit und Sicherheit der verwendeten Pflanzen bzw. Zubereitungen.

Im Studiengebiet konnten 30 betroffene Patienten sowie 9 traditionelle Heiler mit Erfahrung in der Behandlung von Schlafkrankheit für die Studie gewonnen werden. Diese wurden mittels definierter Interview-Protokolle befragt. In nachfolgenden Feldbegehungen wurden die in den Interviews genannten Pflanzen fotografiert, gesammelt und als botanische Belegmuster aufbereitet. Die taxonomische Bestimmung des Pflanzenmaterials erfolgte in Zusammenarbeit mit Botanikern am National Botanical Centre der Universität Agostinho Neto in Luanda.

Von den 30 befragten Patienten gaben 12 (40\%) an, für die Behandlung der Schlafkrankheit allein traditionelle Therapiemassnahmen in Anspruch zu nehmen. Eine zentrale Rolle bei der Entscheidung traditionelle Medizin zu nutzen, spielen die Zugänglichkeit der entsprechenden medizinischen Dienstleistung, die kulturelle Akzeptanz (positive Erfahrungen oder familiäre Gewohnheiten), sowie die Erschwinglichkeit der entsprechenden Therapie. Obwohl es in Angola rund 80-mal mehr traditionelle Heiler als Ärzte gibt, haben nur wenige von ihnen spezifische Erfahrung bei der Behandlung von Schlafkrankheit. Ein wichtiger Grund dafür sind die unspezifischen Symptome in der frühen Phase der Erkrankung, die eine entsprechende Diagnose erschweren. Damit erklärt sich auch, weshalb nur 9 traditionelle Heiler für die Studie gewonnen werden konnten.

Von den Studienteilnehmenden wurden insgesamt 37 verschiedene Pflanzen zur Behandlung der Schlafkrankheit genannt, von denen 30 botanisch vollständig identifiziert werden konnten. Dabei kamen vor allem Wurzeln und Rinden zur Anwendung, die mehrheitlich in Form von Dekokten verabreicht wurden. Die am häufigsten genannte Pflanze ist die Rubiaceae Crossopteryx febrifuga (Afzel. ex G.Don) Benth., welche in weiten Teilen des tropischen Afrikas heimisch ist. Die frischen Blätter der Pflanze werden ausgepresst und als Augen- oder Nasentropfen verabreicht, die Wurzel als Dekokt zubereitet und im Getränk eingenommen. Die Literaturrecherche in wissenschaftlichen Datenbanken ergab, dass für 17 der 30 identifizierten Pflanzen (56\%) in vitro oder in vivo Daten vorliegen, die eine Wirksamkeit bei der Behandlung von Schlafkrankheit bzw. deren Symptome nahelegen. Zu den Pflanzen mit berichteter spezifisch antitrypanosomaler Aktivität gehören der Basilikum Ocimum gratissimum L., der Niembaum Azadirachta indica A. Juss., sowie die Seerose Nymphaea lotus L., auch ägyptische Lotusblume genannt. Dieser Befund bekräftigt unter anderem das Potential des ethnopharmakologischen Ansatzes bei der Suche nach Wirkstoffen. Bei der literaturbasierten Risikoabklärung der verwendeten Pflanzen rückte besonders die Osterluzei Artistolochia gigantea Mart. in den Vordergrund. Die in dieser Gattung allgemein vorkommenden Aristolochiasäuren und deren Metaboliten haben bekanntlich nephrotoxische und karzinogene Eigenschaften. Um auf die entsprechende Problematik hinzuweisen und sichere pflanzliche Alternativen zu definieren, organisieren die Autoren der Studie „feed-back sessions“ mit den Anbietern und Nutzern traditioneller Medizin. Wie in der Schlussfolgerung von Vahekeni und Kollegen/-innen her- 
vorgehoben, repräsentiert diese Studie einen ersten Schritt bei der Validierung von phytotherapeutischen Zubereitungen in der Behandlung von Typanosomiasis in Angola und der Beurteilung ihres potentiellen Nutzens. Weiterführende pharmakologische und toxikologische Untersuchungen der Pflanzen sowie die Etablierung eines Pharmakovigilanzsystems für die eingesetzten traditionellen Therapiemassnahmen sind angezeigt.

Kommentar: Dr. Andreas Lardos (PhD), Fachgruppe Naturstoffchemie und Phytopharmazie, Institut für Chemie und Biotechnologie, ZHAW Life Sciences and Facility Management, Grüentalstrasse 14, 8820 Wädenswil (CH), andreas.lardos@zhaw.ch

\section{Teixidor-Toneu I, Kjesurd K, Kool A. Sweetness Beyond Desserts: The Cultural, Symbolic, and Botanical History of Angelica (Angelica archangelica) in the Nordic Region. J. Ethnobiol., 2020 40(3):289- 304.}

\section{Kommentar}

Maja Dal Cero, Zürich

Die imposante bis zwei Meter hohe Echte Engelwurz Angelica archangelica L. aus der Familie der Doldengewächse, in den Kräuterbüchern der frühen Neuzeit hochgelobte Arzneipflanze, erfährt aktuell in der Phytotherapie wieder vermehrt Beachtung. Die vorliegende Übersichtsarbeit von Teixidor-Toneu et al., 2020, ergänzt pharmakologische und klinische Daten zu AngelikaWurzel-Extrakten und ätherischem Öl um einen interessanten Blick auf den grösseren historischen, geografischen und kulturellen Kontext der Engelwurz-Nutzung in ihrem Ursprungsgebiet. Dabei wird die Bedeutung der Sensorik, des süssen Geschmacks, für die Beziehung zwischen Engelwurz und nordischer Bevölkerung aus historischer und ethnografischer Perspektive vertieft untersucht. Im Laufe der Geschichte hat eine Ausweitung des Verbreitungsgebietes von $A$. archangelica stattgefunden und der Kohlenhydrat-Gehalt insbesondere in den Stängeln wurde teilweise in einem Domestikationsprozess durch Anbau in Gärten und gezielte Selektion erhöht. Gleichzeitig hat sich die Bevölkerung vermehrt auf $A$. archangelica als Nahrungsgrundlage verlassen und damit umgekehrt auch wieder die kulturelle Bedeutung und Wertschätzung beeinflusst. Angelica wurde gewissermassen süsser im Laufe der Zeit - materiell und symbolisch. Gegenwärtig wird Angelica allerdings in der nordischen Küche kaum mehr verwendet.

In der Einleitung fassen die Autorinnen die aktuelle ethnobiologische Diskussion zur Sinneswahrnehmung, die wesentlich die zwischenartlichen Beziehungen prägt, knapp und übersichtlich zusammen. Allgemein kann davon ausgegangen werden, dass Entwicklung und Kontinuität von Mensch-Pflanzen Beziehungen zu Veränderungen in der sensorischen Wahrnehmung von Pflanzen führen kann. Die zitierte Literatur dazu ist eine gute Orientierungshilfe für weitere selbständige Entdeckungen rund um die Bedeutung der Sensorik für ökologische Beziehungen. Insbesondere auch im Hinblick auf die Klassifizierung von Arzneipflanzen anhand ihrer organoleptischen oder sensorischen Qualitäten, wie es seit der Antike bis in die Neuzeit üblich war, sind diese Fragestellungen nach der gegenseitigen Beeinflussung relevant und inspirierend. Die Arbeit von Teixidor-Toneu et al. 2020 geht am Beispiel der Engelwurz vertieft den Fragen nach, seit wann A. archangelica mit der Eigenschaft «süss» in Verbindung gebracht wird und wie diese sensorische Eigenschaft den Wert und die Verwendung im Laufe der Geschichte prägt.

A. archangelica war den antiken Ärzten und Autoren nicht bekannt, da es sich um eine typisch nordische Pflanze handelt mit Hauptverbreitungsschwerpunkt von Grönland über Island und Skandinavien bis nach Ost-Sibirien. Die historische Perspektive der Forschungsfrage wird anhand von schriftlichen Quellen aus drei Epochen beleuchtet: ethnobotanische Literatur des 20. Jahrhunderts, Floren des 18. Jahrhunderts (inkl. der Flora Schwedens von C. Linnaeus, 1745) sowie mittelalterliche Texte aus unterschiedlichen Genren von Gesetztestexten zu Sagenerzählungen. In den zugänglichen mittelalterlichen Texten wurde gezielt nach dem Altnordischen Namen für Angelica, hvo „nn (f.) und hvannir (pl.) gesucht. Um die Ausbreitung von $A$. archangelica seit Wikingerzeiten zu rekonstruieren, wurden zudem das Ortsnamensverzeichnis Norwegens im Hinblick auf Angelica Hinweise (kvann) durchsucht. Für eine Gegenwartsperspektive wurden vier strukturierte Interviews mit Küchenchefs aus der «Neuen Nordischen Küche» in Island und Norwegen geführt. Sie wurden zur aktuellen Verwendung und vor allem zu einer Beschreibung von Geschmack und Geruch von Engelwurz befragt. In der sensorischen Beschreibung erinnert Angelica an aromatischen Sellerie, Süssholz, Dill, Kardamom, Kümmel und wird als süss, blumig, fruchtig, sehr kräftig (powerful) und bitter-süss wahrgenommen.

Alle Daten aus der Literaturrecherche und den Interviews sind in einer Übersichtstabelle mit 210 Einträgen erfasst und lassen unterschiedliche Wissensbestände aus verschiedenen Zeitperioden erkennen. Daraus lassen sich Rückschlüsse auf Veränderungen in der sensorischen Wahrnehmung und in der Nutzung von $A$. archangelica ziehen. Und schliesslich lässt sich damit auch nachverfolgen, wie sich die Beziehung zwischen Mensch und Engelwurz gegenseitig beeinflusst hat.

In vier grösseren Abschnitten wird die Fülle der Resultate ausführlich beschrieben von der sensorischen Wahr- 
nehmung im Laufe der Zeit, über die Bedeutung, zu Fragen der Ausbreitung und Kultivierung und Darstellung der aktuellen Situation. In mittelalterlichen Texten und in der Literatur des frühen 20. Jahrhunderts wird A. archangelica als eine Quelle der Freude dargestellt und gelegentlich explizit als süss beschrieben. Im 19. bis frühen 20. Jahrhundert war A. archangelica das Beste, was einem (Ehren-)Gast als Leckerbissen offeriert werden konnte. Sie war bei der ländlichen Bevölkerung auch ein Festessen zur Mittsommer-Zeit, die Pflanze wurde wild gesammelt und es bestand eine rege Nachfrage. A archangelica spielte als Süssspeise, Gemüse, Gewürz oder zur Aromatisierung von Getränken und als Grundnahrungsmittel seit dem Mittelalter in der Nordischen Medizin, zur Nahrungssicherheit, zu Genuss und kulinarischer Identität eine wichtige Rolle. Die hohlen Stängel fanden Verwendung als solide Röhren und die gefüllten Blattstiele mit ihrem höheren Kohlenhydratgehalt als Nahrungsmittel. Es ist nicht bekannt wann genau und wo die Kultivierung von $A$. archangelica begann, doch finden sich in verschiedenen älteren nordischen Gesetzestexten Hinweise darauf, dass Angelica ein wertvoller landwirtschaftlicher Rohstoff war. In den letzten Jahrzehnten gibt es auf den Färöern ein steigendes Interesse an der Wiederbelebung der ursprünglichen Engelwurz-Gärten und in Norwegen hat man begonnen $A$. archangelica erneut zu züchten.

In der Diskussion vermuten die Autorinnen, dass die breite Palette an Nutzungsmöglichkeiten (ausser Nahrung und Medizin auch als Amulett gegen Zaubersprüche und als Glücksbringer, als Spielzeug, Ersatz an Stelle von Tabak, als Räucherwerk, als Musikinstrument u.a.) die Ausbreitung und teilweise Domestizierung von A. archangelica begünstigt haben.

Zusammenfassend wird die süsse Qualität von A. archangelica nicht ausschliesslich als eigentliche Geschmacksrichtung interpretiert, sondern auch im kulturellen Zusammenhang mit der angenehmen Erfahrung der Ernte und von (Mittsommer-)Festessen gesehen.

Sensorische Wahrnehmungen, die sich im Laufe der Zeit durchaus verändern können, beeinflussen offensichtlich neben pharmakologischen Wirkungen und klinischen Wirksamkeiten sowie regulatorischen und ökonomischen Rahmenbedingungen, wie das Potential einer Arzneipflanze genutzt wird. Die vorliegende Arbeit ermöglicht damit einen erfrischend erweiterten Blick auf $A$. archangelica, wie er auch für andere Pflanzen, die eine lange Nutzungsgeschichte als Arznei- oder Nahrungspflanze aufweisen, wünschenswert wäre.

Kommentar: Dr.sc.nat. Maja Dal Cero, Institut für Systematische und Evolutionäre Botanik und Botanischer Garten, Universität Zürich, Zollikerstrasse 107, 8008 Zürich, Schweiz, maja.dalcero@uzh.ch

\section{Glover DM. Traditional Medicines in a Global Economy: Resource Sustainability and Resilience in the Traditional Tibetan Medical Practice of Ingredient Substitution. Hum Ecol. 2021; 49:33-42.}

\section{Kommentar}

Franz Huber, Winterthur

Nachhaltigkeit und Resilienz sind zwei zentrale Begriffe der sozioökologischen Forschung, und es sind Konzepte, die auch im Zusammenhang mit Arzneipflanzen auf verschiedenen Ebenen angewandt werden können. Nachhaltigkeit ist das Prinzip der dauerhaften Aufrechterhaltung eines Systems, und Resilienz kann definiert werden als die Fähigkeit eines Systems, sich an plötzliche Veränderungen anzupassen und wieder zu seinen grundlegenden Funktionen zurückzukehren. Während die Forschung zu phytotherapeutischen Arzneimittel schon immer auf das komplexe Zusammenspiel eines Vielstoffgemisches mit verschiedenen biochemischen Strukturen (und damit gewissermassen auf die Nachhaltigkeit und Resilienz auf der biochemischen Ebene) fokussiert haben, gelangen mit den Herausforderungen im Bereich Klimawandel und globaler Wertschöpfungsketten immer mehr auch ökologische und sozioökonomische Fragen wie die Herkunft und Produktion von pflanzlichen Arzneimitteln in den Fokus sowohl von Forschung als auch von Konsument*innen.

Der vorliegende Artikel von Denise M. Glover in der Zeitschrift Human Ecology bietet eine gute Einführung in diese weite Thematik am Beispiel der Substitution von Zutaten in traditionellen Tibetischen Rezepturen. Weltweit stehen natürliche Ressourcen, und insbesondere wild gesammelte Arzneipflanzen, unter Druck. Während die Nachfrage zunimmt und in Folge globaler Trends häufig stark fluktuiert, machen sich auf Seiten des Angebots immer mehr die Auswirkungen des Klimawandels aber auch die Abhängigkeiten von lokalen Produzenten und Wildsammlern bemerkbar: Sammelmengen nehmen ab, die Qualität der Produkte schwankt, die Einkommensbasis von ganzen lokalen Gemeinden ist gefährdet. Mit anderen Worten: Die Nachhaltigkeit der Produktion pflanzlicher Arzneimittel ist oft nicht gegeben. Im vorliegenden Artikel argumentiert die Autorin nun, dass durch die traditionelle Praxis der Substitution von Zutaten in der traditionellen tibetischen Medizin (und anderen traditionellen Medizinsystemen) eine adaptive Strategie vorhanden ist, die solchen Druck reduzieren kann, indem problematische Zutaten durch alternative Bestandteile ersetzt werden und sich dadurch die Nachhaltigkeit und Resilienz des Gesamtsystems erhöhen. Ein interessanter Punkt dabei ist die Feststellung, dass durch diese Praxis der Substitution nicht nur eine Anpassung an ändernde Rahmenbedingungen stattfindet, sondern dass damit in- 
härent auch eine Innovationfähigkeit des Medizinsystems verbunden ist. Dieser Punkt macht diese Publikation über die Tibetische Medizin hinaus auch für die Leserschaft in Europa interessant und bedenkenswert, sieht die Autorin doch in den gegenwärtigen Prozessen der immer strikteren Standardisierungen und Validierungen im Bereich der pflanzlichen Arzneimittel ein zunehmendes Hindernis für Innovationen und Anpassungen an sich ändernde Bedingungen. Vor diesem Hintergrund ist die Diskussion, wie sich Standardisierung und Sicherheit auf der einen Seite und eine gewisse adaptive Flexibilität auf der anderen Seite die Waage halten können, sicher noch nicht abgeschlossen.

Kommentar: Dr. Franz Huber, Schweizerische Medizinische Gesellschaft für Phytotherapie und Institut für Systematische und Evolutionäre Botanik, Universität Zürich, Zollikerstrasse 107, 8008 Zürich, Schweiz, f.huber@smgp.ch

\section{Sardar Muhammad Rafique Khan, Tanveer Akhter and Mumtaz Hussain. Ethno-veterinary practice for the treatment of animal diseases in Neelum Valley, Kashmir Himalaya, Pakistan. Plos One 16(4): e0250114. https://doi.org/10.1371/journal. pone.0250114.}

\section{Kommentar \\ Michael Walkenhorst, Frick}

Ethnoveterinärmedizinische Erkenntnisse bestehen nach wie vor aus einem weltweit losen Flickenteppich von Informationen. Wann immer dieser Flickenteppich ein Stück dichter wird ist das ein wichtiger Schritt (und eine Freude für den Kommentator), insbesondere, wenn so intensiv gearbeitet wurde, wie in der vorliegenden Studie. Im Upper Neelum Valley, einem Tal in der nordöstlichen Region Kaschmir im Nordosten Pakistans wird überwiegend selbstversorgende Weidewirtschaft durch halbnomadisch lebende Hirten betrieben. Im Verlauf von 3 Jahren wurden hier durch das Forscher*innenteam 73 Frauen und 53 Männer interviewt, wobei darauf geachtet wurde insbesondere ältere und fachlich versierte Gesprächspartner*innen zu finden. Teilweise wurden die Gesprächspartner*innen auch zu den Sammelorten der Arzneipflanzen begleitet oder es wurde ihnen bei der $\mathrm{Zu}$ bereitung der Präparate zugeschaut. Vor allem ältere Frauen hatten ein reiches und häufig angewendetes Wissen über den Einsatz der lokalen Flora zur traditionellen Behandlung der Krankheiten ihrer Haustiere.

Insgesamt wurden in den Interviews 39 Pflanzenarten aus 21 Familien zur Behandlung von Krankheiten von
Rindern, Büffeln, Schafen und Ziegen beschrieben. Zudem wurde auch noch der verwendete Pflanzenteil und die Zubereitung erfasst. Mit insgesamt 7 Pflanzenarten waren die Knöterichgewächse (Polygonacea) die artenreichste Familie unter den traditionell eingesetzten Arzneipflanzen. Im Hinblick auf die therapeutische Intention des Arzneipflanzeneinsatzes wurden 21 Einsatzbereiche unterschieden, darunter Erkrankungen des Magen-Darm-Traktes, respiratorische Erkrankungen und Parasitosen. Zahlreiche Arzneipflanzenzubereitungen wurden aber auch zur allgemeinen Stärkung geschwächter Tiere («tonic») oder gezielt zur Stärkung von Muttertieren unmittelbar nach der Geburt verabreicht («post-delivery treatment»). Berücksichtigt man, dass viele der eingesetzten Pflanzenarten zur natürlichen Flora des Untersuchungsgebiets zählen und damit vermutlich zu einem grossen Teil für weidende Tieren zugänglich sind, befinden wir uns beim Indikationsgebiet der allgemeinen Stärkung mitten im Überschneidungsbereich zwischen einer gesundherhaltenden Futtervielfalt mit der Möglichkeit zur Selbstmedikation von Tieren und traditioneller gezielter fürsorglicher Anwendung wirkstoffreicher Pflanzen durch Tierhalter*innen.

Die Verabreichung der überwiegend frischen oder gekochten Pflanzenteile fand zum grössten Teil oral statt. Besonders häufig und bei unterschiedlichen Indikationen wurden die zur Familie der Korbblütler und zur Gattung der Alpenscharten gehörende Indische Kostuswurzel («Saussurea lappa» der aktuell anerkannte wissenschaftliche Name ist laut dem Medicinal Plant Name Service allerdings Aucklandia costus Falc. [1]), die beiden zur Familie der Knöterichgewächse gehörenden Ampferarten Rumex acetosa L. (Sauerampfer) und Rumex nepalensis Spreng sowie die zur Familie der Lippenblütler gehörende Thymianart Thymus linearis Benth eingesetzt. Hier gibt es interessante Parallelen zu ethnoveterinärmedizinischen Forschungsergebnissen aus dem Alpenraum [2], wo ebenfalls der Einsatz der Gattungen Ampfer und Thymiane häufig beschrieben ist. Es wäre für die Zukunft interessant, würde sicher das gegenseitige Verständnis fördern und vielleicht auch neue Möglichkeiten eröffnen, zu versuchen ethnoveterinärmedizinische Erkenntnisse einer Region in eine andere klimatisch vergleichbare aber regional weit auseinanderliegende Region durch die Suche nach Pflanzen aus derselben Gattung zu «übersetzen».

Während die Wurzel der Indischen Kostuswurzel überwiegend zur allgemeinen Stärkung eingesetzt wird, ist die Indikation für die Ampferarten die gezielte Stärkung von Muttertieren nach der Geburt. Auch bei diesen beiden Arten kommt die Wurzel zum Einsatz. Das Kraut von Thymus linearis Benth hat sein Hauptindikationsgebiet in der Behandlung von Erkrankungen des Magen-Darm-Traktes. Bemerkenswert und wichtig ist, 
dass die Autoren für jede erfasste Pflanzenart den Gefährdungsgrad ausgewiesen haben. Sowohl die Indische Kostuswurzel als auch Thymus linearis Benth gelten als bedroht. So wichtig die ethnoveterinärmedizinische Forschung ist, so wichtig ist auch, dass diese die Bedrohung für die wild gesammelte Pflanzenarten kritisch reflektiert.

Noch aus vielen Regionen dieser Erde fehlen ethnoveterinärmedizinische Erkenntnisse. Hoffen wir, dass sich noch so manche Forscher*innengruppe auf den Weg macht Stoff in den Flickenteppich einzuweben.

\section{Literatur}

1 Stucki K, Dal Cero M, Vogl CR, Ivemeyer S, Meier B, Maeschli A, Hamburger M, Walkenhorst M. Ethnoveterinary contemporary knowledge of farmers in pre-alpine and alpine regions of the Swiss cantons of Bern and Lucerne compared to ancient and recent literature - is there a tradition? J. Ethnopharmacol. 2019;234:225-244.

Kommentar: Dr. Michael Walkenhorst, Fachgruppe Tiergesundheit, Forschungsinstitut für biologischen Landbau (FiBL), Ackerstrasse 113, Postfach 219, 5070 Frick, Schweiz, michael.walkenhorst@fibl.org 